Des expériences plus récentes effectuées par KronenwetT et coll. [41] donnèrent lieu à la courbe de destruction des $\mathrm{Br}$. abortus, représentêe dans le graphique 311 .

En résumé, on peut donc constater, en partant des résultats des recherches mentionnées, que le chauffage du lait à des températures suffisantes pour rendre la phosphatase inactive offre une garantie certaine de destruction des Brucella.

(A suivre.)

\title{
NOUVEAUX ESSAIS INDUSTRIELS DE SUPERCENTRIFUGATION BACTÉRIENNE DU LAIT (1)
}

\author{
par \\ PAUl SIMONART, Roger POFFÉ et Alois WUYTACK \\ Université de Louvain \\ Station de Recherches laitières, Héverlé-Louvain
}

\section{Introduction}

Une première série d'essais industriels de supercentrifugation bactérienne, qui portait sur quelque 500.000 litres de lait, a montré que l'ón pouvait, par cette méthode, éliminer près de $93 \%$ des bactéries que contenait originellement le lait [1]. Outre que ces expériences aient prouvé l'intérêt et la possibilité d'application de la supercentrifugation bactérienne, ils ont aussi mis en évidence que les résultats obtenus à l'échelle industrielle, tout en étant satisfaisants, étaient moins bons que ceux qui avaient antérieurement été observés à l'échelle semi-industrielle [2].

Les expériences industrielles ont done été poursuivies dans le but de relever le taux d'élimination des bactéries et de le ramener au voisinage de celui qui est obtenu semi-industriellement. C'est dans ce but que certaines modifications ont été apportées dans la structure intérieure $\mathrm{du}$ bol, tandis qu'en même temps le matériel était rigoureusement désinfecté.

Les conditions expérimentales sont celles appliquées précédemment [1] : le centrifugeur utilisé possède une capacité de $3.000 \mathrm{l}$./h. et peut développer une force centrifuge d'environ $9.000 \mathrm{~g}$. Tous les essais ont été faits avec un bol pourvu de deux ouvertures de $0,4 \mathrm{~mm}$. Quant aux méthodes de contrôle utilisées, ce sont aussi celles qui ont été appliquées pour les essais antérieurs.

(1) Travail effectué sous les auspices de l'I.R.S.I.A. (Institut pour l'Encouragement de la Recherche Scientifique dans l'Industrie et l'Agriculture), dans le cadre des travaux du Comité pour l'Etude du Lait. 


\section{Résultats et discussion}

Le tableau I donne les résultats obtenus pour une trentaine d'essais qui portent sur environ 200.000 litres de lait. On y remarque que dans plusieurs expériences, le pouvoir séparateur est voisin ou même supérieur à $98 \%$. La moyenne des chiffres obtenus donne un pouvoir séparateur moyen de $96,65 \%$ et de $97,18 \%$ selon qu'on l'exprime en colonies ou en cellules bactériennes. Ceci marque une sensible amélioration par rapport aux rendements des expériences précédentes.

Il est à remarquer que la température de préchauffage de $72^{\circ}$ donne un lait dont l'épreuve de la phosphatase indique de 4 à 6 Unités Lovibond. Les conditions thermiques appliquées n'équivalent donc pas à une pasteurisation, bien que le grand écart observé entre le nombre de colonies et le nombre de cellules du lait préchauffé prouve qu'elles exercent une nette action microbicide.

Le bol de supercentrifugeur étant perforé de deux orifices de $0,4 \mathrm{~mm}$, il s'en échappe de façon continue une certaine quantité de lait. Cette "perte" de lait est de 90 litres par heure ce qui correspond à $3 \%$. Mais lorsque c'est du lait non homogénéisé que l'on soumet à la supercentrifugation, le lait qui s'échappe par les orifices phériphériques du bol ne contient guère que $0,1 \%$ de matière grasse. Ce qui revient à dire que pour la fabrication de 97 litres de lait entier supercentrifugé, il y a une perte de 3 litres de lait écrémé.

Ceci implique encore que, dans la standardisation, il y a lieu de tenir compte de cette concentration en matière grasse qui est de 100/97. Lorsque l'on traite du lait semi-homogénéisé, le lait qui s'échappe à la périphérie contient $0,8 \%$ de matière grasse, ce qui correspond à une valeur commerciale qui est inférieure à celle d'un litre de lait entier.

Bien que cette " perte" ne soit pas un empêchement économique d'application du procédé, il y a cependant lieu de noter d'abord qu'elle pourrait encore être réduite par réduction des dimensions des orifices en question, et ensuite, que ce lait peut encore trouver une destination dans l'alimentation du bétail, soit après pasteurisation, soit après dessication.

\section{Résumé}

Les résultats exposés dans cette note montrent que la supercentrifugation bactérienne du lait appliquée à l'échelle industrielle, permet d'éliminer $97 \%$ des bactéries que contenait le lait préchauffé. Cette note traite encore de l'aspect quantitatif du lait qui s'échappe par les orifices minuscules forés dans la paroi du bol du centrifugeur. 


\section{TABLEAU I}

ESSAIS INDUSTRIELS DE SUPERCENTRIFUGATION BACTÉRIENNE (3.000 L/H A 9.000 G.). LA TENEUR EN BAGT.RIES DU LAIT GENTRIFUGÉ EST EXPRIMÉ EN POURGENTAGE DE LA TENEUR DU LAIT PRÉGHAUFFé

\begin{tabular}{|c|c|c|c|c|c|}
\hline $\begin{array}{l}N^{\circ} \mathrm{de} \\
\text { l'essai }\end{array}$ & $\begin{array}{c}\text { Température } \\
\text { de pré- } \\
\text { chauffage } \\
{ }^{\circ} \mathrm{C}\end{array}$ & $\begin{array}{l}\text { Nombre } \\
\text { bact. p } \\
\text { préchauffé } \\
10^{3} / \mathrm{ml} \text {. }\end{array}$ & $\begin{array}{l}\text { colonies } \\
\text { le lait } \\
\text { centrifugé } \\
\%\end{array}$ & $\begin{array}{c}\text { Nombre de o } \\
\text { (Breed) p } \\
\text { préchauiffé } \\
10^{3} / \mathrm{ml} .\end{array}$ & $\begin{array}{l}\text { ules bact. } \\
\text { le lait } \\
\text { centrifug } \\
\%\end{array}$ \\
\hline $128 \mathrm{a}$ & 72 & 51 & 3,5 & 5.460 & 3,6 \\
\hline $\mathbf{b}$ & 72 & 33 & 2,4 & 6.435 & 3,3 \\
\hline $130 \mathrm{a}$ & 72 & 62 & 2,1 & 1.723 & 2,1 \\
\hline b & 72 & 70 & 1,7 & 1.755 & 3,0 \\
\hline $132 \mathrm{a}$ & 72 & 79 & 4,4 & 1.840 & 2,1 \\
\hline b & 72 & 53 & 1,6 & 3.614 & 2,6 \\
\hline $134 \mathrm{a}$ & 72 & 48 & 4,0 & & \\
\hline $\mathbf{b}$ & 72 & 47 & 5,2 & 1.800 & 1,7 \\
\hline $136 \mathrm{a}$ & 72 & 16 & 5,6 & 663 & 1,6 \\
\hline b & 72 & 20 & 3,4 & & \\
\hline $138 \mathrm{a}$ & 72 & 25 & 2,6 & 839 & 2,3 \\
\hline $\mathbf{b}$ & 72 & 24 & 2,5 & 988 & 5,3 \\
\hline $140 \mathrm{a}$ & 72 & 18 & 1,5 & 299 & 4,5 \\
\hline b & 72 & 16,7 & 1,6 & 227 & 3,4 \\
\hline 142 a & 72 & 22 & 4,9 & 3.100 & 2,4 \\
\hline b & 72 & 29,7 & 4,4 & 3.009 & 2,1 \\
\hline $144 \mathrm{a}$ & 72 & 26 & 5,3 & 2.152 & 3,6 \\
\hline b & 72 & 43,5 & 3,6 & 1.703 & 3,2 \\
\hline $146 \mathrm{a}$ & 72 & & & 2.730 & 2,3 \\
\hline $\mathrm{b}$ & 72 & & & 2.652 & 1,1 \\
\hline $150 \mathrm{a}$ & 72 & 62 & 2,4 & & \\
\hline $153 \mathrm{a}$ & 72 & 51 & 4,2 & 1.410 & 1,3 \\
\hline $\mathrm{b}$ & 72 & 64 & 2,8 & & \\
\hline $155 \mathrm{a}$ & 72 & 90 & 3,1 & 871 & 3,7 \\
\hline $\mathrm{b}$ & 72 & 89 & 3,8 & 845 & 4,9 \\
\hline $157 \mathrm{a}$ & 72 & 47 & 2,0 & 1.690 & 1,9 \\
\hline $\mathrm{b}$ & 72 & 42 & 2,1 & 1.404 & 2,6 \\
\hline $159 \mathrm{a}$ & 72 & 7,1 & 4,0 & 1.131 & 2,7 \\
\hline b & 72 & 9,4 & 3,4 & 1. 287 & 2,1 \\
\hline $166 \mathrm{a}$ & 72 & 50 & 3,1 & 2.067 & 2,3 \\
\hline $168 \mathrm{a}$ & 72 & 64 & 4,3 & 3.315 & 2,3 \\
\hline $170 \mathrm{a}$ & 72 & 44 & 3,1 & 9.360 & 4,6 \\
\hline $172 a$ & 74 & 74 & 2,8 & 8.450 & 3,5 \\
\hline $174 \mathrm{a}$ & 73 & 158 & 5,8 & 4.840 & 2,6 \\
\hline
\end{tabular}

(Les auteurs expriment leurs remerciements à la Compagnie Lacsoons et à la Société $\mathrm{Al}$ fa Laval pour leur précieuse collaboration matérielle à ces essais.) 


\section{BIBLIOGRAPHIE}

[1] P. Simonart, R. Poffe et A. Wuytack. Neth. Milk and Dairy Journal, 10, 1956, 163.

[2] P. Simonart et G. Debeer. Neth. Milk and Dairy Journal, 8, 1954, 163.

\section{L'AVENIR DE LA MÉTHOdE GERBER (1) par JEAN PIEN}

Le dosage de la matière grasse du lait liquide peut s'effectuer à l'aide de plusieurs méthodes qui se répartissent en deux groupes :

- Méthodes pondérales, dont le type est la méthode RöseGottlieb, qui a été récemment admise par la Fédération Internationale de Laiterie comme méthode standard internationale [1];

- Méthodes volumétriques, dont le type est la méthode Gerber dont nous avons montré la supériorité sur la méthode Babcock et sur toutes les autres méthodes volumétriques [2].

La méthode acido-butyrométrique de Gerber, universellement connue et chaque jour appliquée dans des milliers d'entreprises de laiterie, est une méthode industrielle rapide dont la fidélité et l'exactitude peuvent être amenées à un niveau très voisin de celles des méthodes pondérales - voire même identique - sous la réserve de s'entourer de certaines précautions dans la mesure de la prise d'essai, dans la lecture du résultat et de disposer de butyromètres correctement gradués [2].

Cependant, la méthode Gerber a traversé ces dernières années une "crise" grave. Son exactitude a été, en effet, mise en cause par de nombreux auteurs. Etant donné la très grande importance prise par cette méthode dans la pratique industrielle courante, nous voulons faire le point de la question et diseuter notamment de cette inexactitude, des remèdes qui y ont été apportés dans divers pays, des remèdes qu'il convient à notre avis d'appliquer, des bases correctes de la graduation des butyromètres à lait, des autres conditions d'exactitude de la méthode Gerber ainsi que de ses possibilités et de son avenir.

$$
\text { *** }
$$

\section{Inexactitude de la méthode Gerber classique}

Le point fondamental sur lequel repose la méthode Gerber est le mode de graduation du butyromètre. Or, le butyromètre Gerber

(1) Communication au XIVe Congrès International de Laiterie (Rome, Septembre 1956) 